University of Wollongong

Research Online

Faculty of Social Sciences - Papers (Archive) Faculty of Arts, Social Sciences \& Humanities

2013

Prestimulus delta and theta determinants of ERP responses in the Go/ NoGo task

Frances M. De Blasio

University of Wollongong, francesd@uow.edu.au

Robert J. Barry

University of Wollongong, rbarry@uow.edu.au

Follow this and additional works at: https://ro.uow.edu.au/sspapers

Part of the Education Commons, and the Social and Behavioral Sciences Commons

Research Online is the open access institutional repository for the University of Wollongong. For further information contact the UOW Library: research-pubs@uow.edu.au 


\title{
Prestimulus delta and theta determinants of ERP responses in the Go/NoGo task
}

\begin{abstract}
Ongoing low-frequency EEG activity has long been associated with ERP components and their cognitive processing interpretations, yet few studies have directly investigated the prestimulus low-frequency EEGERP relationships, particularly within the auditory domain. The present study assessed the delta (1-3 Hz) and theta $(4-7 \mathrm{~Hz})$ bands individually, and their prestimulus influence on five subsequent components (P1, N1, P2, N2, and P3) within an equiprobable auditory Go/NoGo paradigm. At the nine central sites, accepted trials were sorted according to their ascending vertex prestimulus spectral band amplitude, and ERPs were derived from the upper and lower sorted thirds. The within-subjects analyses included amplitudes and latencies for both Go and NoGo responses, and Go response speed. Only component amplitudes showed effects of high/low prestimulus EEG level. Delta globally modulated the five components - all amplitudes were more positive with high prestimulus delta, regardless of stimulus condition. Theta did not influence P1, but inversely modulated P2 and P3 regionally, and produced stimulus-specific effects in N1, N2, and P3. Low prestimulus theta produced greater NoGo N2 and Go P3, and reduced NoGo P3 responses, each of these suggesting appropriately enhanced cognitive processing. Taken together, these effect patterns differentially implicate prestimulus delta and theta band activity in the determination of ERP component amplitudes and the cognitive processing associated with them.
\end{abstract}

\section{Keywords}

erp, responses, go, determinants, nogo, prestimulus, task, theta, delta

\section{Disciplines}

Education | Social and Behavioral Sciences

\section{Publication Details}

De Blasio, F. M. \& Barry, R. J. (2013). Prestimulus delta and theta determinants of ERP responses in the Go/NoGo task. International Journal of Psychophysiology, 87 (3), 279-288. 
Prestimulus delta and theta determinants of ERP responses in the Go/NoGo task.

Frances M. De Blasio and Robert J. Barry*

Centre for Psychophysics, Psychophysiology, and Psychopharmacology; Brain \& Behaviour Research Institute; and School of Psychology,

University of Wollongong,

Wollongong NSW 2522,

Australia

*Corresponding author

Email: rbarry@uow.edu.au

Phone: +61242214421

Fax: +61 242214421 


\begin{abstract}
Ongoing low-frequency EEG activity has long been associated with ERP components and their cognitive processing interpretations, yet few studies have directly investigated the prestimulus low-frequency EEG - ERP relationships, particularly within the auditory domain. The present study assessed the delta (1-3 Hz) and theta (4-7 Hz) bands individually, and their prestimulus influence on five subsequent components (P1, N1, P2, N2, and P3) within an equiprobable auditory Go/NoGo paradigm. At the nine central sites, accepted trials were sorted according to their ascending vertex prestimulus spectral band amplitude, and ERPs were derived from the upper and lower sorted thirds. The within-subjects analyses included amplitudes and latencies for both Go and NoGo responses, and Go response speed. Only component amplitudes showed effects of High/Low prestimulus EEG level. Delta globally modulated the five components - all amplitudes were more positive with high prestimulus delta, regardless of stimulus condition. Theta did not influence P1, but inversely modulated P2 and P3 regionally, and produced stimulus-specific effects in N1, N2, and P3. Low prestimulus theta produced greater NoGo N2 and Go P3, and reduced NoGo P3 responses, each of these suggesting appropriately enhanced cognitive processing. Taken together, these effect patterns differentially implicate prestimulus delta and theta band activity in the determination of ERP component amplitudes and the cognitive processing associated with them.
\end{abstract}

Keywords: EEG/ERP, ERP genesis, delta, theta, auditory Go/NoGo, cognitive processing 


\section{Introduction}

When presented with a stimulus, there is a robust association between our ongoing electroencephalographic (EEG) activity and the amplitude and latency of the event-related potential (ERP) elicited. Across a range of auditory tasks (our focus here), two analytic approaches have consistently shown links between the poststimulus EEG activity of the lowfrequency bands, here referring to delta $(\sim 0.5-3.5 \mathrm{~Hz})$ and/or theta $(\sim 3.5-7 \mathrm{~Hz})$, and the concomitant ERP components. Spectral analysis of the wide-band ERPs has shown dominant poststimulus amplitude/power contributions from one or both of these low-frequency bands (Başar et al., 1984; Başar-Eroglu et al., 1992; Başar-Eroglu \& Demiralp, 2001; Başar-Eroglu et al., 2001; Spencer \& Polich, 1999), particularly within the latency window of the endogenous components (> 250 ms). Coinciding peak characteristics are noted between the event-related oscillations (EROs: the poststimulus phase-locked EEG activity within a set band, typically derived via filtering) of the delta and/or theta band and the P3 (Başar \& Stampfer, 1985; Karakaş et al., 2000; Stampfer \& Başar, 1985; Yordanova \& Kolev, 1998), amongst other components. Also, N1 and N2 amplitudes are topographically enhanced where delta EROs are minimal, and theta EROs are maximal (Barry, 2009).

Like the endogenous ERP components themselves, poststimulus activity in the delta and theta bands appears sensitive to stimulus and/or paradigm manipulation. For instance, in comparison to equiprobable Go stimuli, NoGo stimuli provoke greater and prolonged poststimulus spectral power enhancement in the low-frequency bands, particularly within the endogenous period (Barry, 2009). For oddball targets vs. nontargets, EROs amplitudes are enhanced in the delta and theta bands, the delta EROs peak is delayed, and the theta EROs amplitude enhancement is sustained (Stampfer \& Başar, 1985). With increased task complexity, poststimulus increases are noted in the spectral amplitudes of the wide-band ERPs, and also in the EROs amplitudes, with these effects occurring across the broad poststimulus period for delta, and the endogenous-specific period for theta (Başar-Eroglu, et al., 1992; Başar-Eroglu \& Demiralp, 2001; Başar-Eroglu et al., 
2001). Prolonged enhancement of the EROs in theta has also been reported for task compared to no-task conditions (Kolev \& Schürmann, 1992). Parallel increases in low-frequency spectral power and P3 amplitudes have been reported with manipulations of stimulus repetition, probability, and response requirements (Spencer \& Polich, 1999). Similarly, theta EROs and P3 amplitudes have shown simultaneous increases for oddball targets requiring a response, when compared to passive task conditions (Yordanova \& Kolev, 1998).

The superposition of EROs across various bands, predominantly those of the low frequencies, was proposed to closely approximate the ERP (Başar \& Stampfer, 1985; Stampfer \& Başar, 1985). This has been supported, with EROs in the delta and theta bands reported to directly predict the N2 and P3 component amplitudes, accounting for 57-97\% of their variation at midline sites across a range of paradigms (Karakaş et al., 2000). Moreover, a direct correlation has been found between single trial endogenous theta EROs and P3 amplitudes (Yordanova \& Kolev, 1998), and as mentioned earlier, the topographies of the delta and theta EROs largely determine the N1 and N2 amplitude topographies (Barry, 2009). Overall, poststimulus EEG activity in the low-frequency bands appears to be closely related to ERP component characteristics.

Early investigations by Başar and colleagues also noted the occurrence of prestimulus spectral amplitude - EROs enhancements in the delta band following stimulation (Stampfer \& Başar, 1985), and in the theta band for omitted stimuli (Başar et al., 1984) in some trials. These studies were exploratory, with no statistical analyses reported, and methodological concerns have been raised regarding their digital filtering (Barry et al., 2000). However, pre-post stimulus EEG spectral power increases in one or both low-frequency bands have since been confirmed using improved signal processing techniques including Fast Fourier Transform (FFT) algorithms (Barry, 2009; Yordanova \& Kolev, 1998). Direct correlations have also been noted between prestimulus spectral amplitudes and poststimulus EROs amplitudes in the theta band at the single trial level (Yordanova \& Kolev, 1998). Thus the low-frequency EEG activity outside the poststimulus period is considered to contribute to the ERP. For instance, the low-frequency spectral power of 
the 'background’ EEG, recorded separately either before or after the ERP paradigm, is directly correlated with the size of the N1 for both target and standard oddball stimuli (Intriligator \& Polich, 1995), and with target oddball P3 amplitude (Intriligator \& Polich, 1994, 1995; Polich, 1997), when both the EEG and ERPs are recorded with open eyes. This 'background' EEG - P3 correlation has been consistent across a range of target probabilities (Intriligator \& Polich, 1994), and across a broad age range (Polich, 1997). However, given the dynamic nature of the ongoing EEG fluctuations, we suggest that this non-task period is suboptimal to elucidate the most influential EEG determinants of the ERP - rather, the EEG brain state immediately preceding stimulus onset would seem more salient.

There remains some debate surrounding the underlying mechanism(s) involved in ERP generation, specifically the nature and extent of the EEG - ERP contribution. Of the two major models of ERP genesis, the evoked model describes the ERP as the sum of distinct event induced activity overlaying, and independent of, the ongoing EEG following stimulus onset (Barry, 2009; Jervis, Nichols, Johnson, Allen, \& Hudson, 1983; Klimesch, Sauseng, Hanslmayr, Gruber, \& Freunberger, 2007; Min et al., 2007; Sauseng et al., 2007). Alternatively, the phase-reset model implicates the phase spectra of the ongoing EEG as a determining factor in ERP genesis - that is, event specific phase reorganization and alignment occurs in specific frequencies of the ongoing EEG following stimulus onset (Barry, 2009; Klimesch et al., 2007; Min et al., 2007; Sauseng et al., 2007). Each model provides a differential account of the EEG - ERP relationship, but implicit within each model is the influence of the EEG activity immediately prestimulus (Başar et al., 1984; Başar \& Stampfer, 1985; Min et al., 2007). We are not primarily concerned here with the mechanisms underlying each model; instead our interest is in their empirically assessable expression.

Remarkably few studies in the auditory domain have assessed the within-task prestimulus EEG - ERP relationships for the low-frequency bands. Rahn and Başar (1993) reported withinsubject N1-P2 amplitude enhancements for low prestimulus theta trials. However, each stimulus 
presentation was contingent upon the spectral amplitude within each participant's theta band, and the possibility of biofeedback-type confounds has since been raised (Barry et al., 2000). Romani et al. (1988) found within-subject lengthening of N1 latencies across four ERPs derived from trials with increasing proportions of prestimulus combined delta and theta activity. Across these levels, participants with greater prestimulus low-frequency spectral amplitudes showed lower N1-P2 amplitudes and greater N1 latencies. The above two studies employed single-stimulus paradigms, the first a passive task, and the second a slow count task, providing only limited generalisability. Since then, Lazzaro et al. (2001) have reported increased oddball P2 amplitude, N1 and P3 latencies, and reaction time (RT), and decreased N2 amplitudes in an adolescent AD/HD population, who also had increased prestimulus spectral power within the theta band, compared to age- and sex-matched controls. Taken together, the findings across the three studies implicate prestimulus EEG activity in the delta and theta bands as important determinants of the N1, P2, N2, and P3 components and response performance, yet they remain to be systematically verified and extended to incorporate additional stimulus and task conditions.

In addition to the AD/HD field, low-frequency EEG and ERP covariations are mentioned in diverse literatures. From childhood to adolescence, developmental studies generally report reductions in delta and theta band power (Barry \& Clarke, 2009), latency decreases for one or more of the N1, N2 and/or P3 components (Kolev et al., 2001), amplitude reductions in the NoGo N2, and increases in the P3 (Segalowitz et al., 2010). Delta band activity typically has limited and mixed findings within the meditation literature, although increases in theta power across differing meditation types, particularly transcendental, are generally noted, as is evidence for meditationbased P3 latency decreases (Cahn \& Polich, 2006). Such covariations across literatures have prompted cognitive-processing interpretations of the functional significance of the low-frequency EEG bands, theta more so than delta.

Activity in the delta band has been associated with decision-making, signal detection, and/or stimulus matching, primarily due to the nature of its EROs amplitude enhancements with 
increased task complexity across paradigms (Başar et al., 2000, 2001; Başar-Eroglu et al., 1992; Başar-Eroglu \& Demiralp, 2001; Başar-Eroglu et al., 2001). Theta has been implicated in several cognitive processes, most notably in relation to attention, due to its pattern of prolonged and/or endogenous EROs amplitude enhancement with increased task complexity across paradigms (Başar et al., 2000, 2001; Başar-Eroglu et al., 1992; Başar-Eroglu \& Demiralp, 2001; Başar-Eroglu et al., 2001); as well as memory (Klimesch et al., 2007), with reduced prestimulus, and augmented poststimulus, spectral power in theta differentiating good from poor memory task performers (Klimesch, 1999). Moreover, the combined low-frequency EEG contributions to working memory appear important, given the selectively distributed nature of the EROs response across bands (Başar et al., 2000, 2001).

Our study aimed to investigate the nature and extent of the prestimulus delta and theta determinants of five ERP components (P1, N1, P2, N2, and P3) in an equiprobable auditory Go/NoGo paradigm. This task was selected to decrease stimulus probability and inhibition-related confounds (Lavric et al., 2004). We decided to assess the spectral amplitude of the prestimulus EEG at the vertex as this is the centre of the nine scalp sites where ERPs were to be examined. For each EEG band, two levels of within-subject prestimulus activity (High vs. Low) were used to investigate amplitude and latency effects in each ERP component and stimulus condition separately. Go RT was also included, allowing the assessment of prestimulus low-frequency EEG effects on behavioural performance. No formal hypotheses were made for either band across the measured outcomes, although speculations were extrapolated from the limited reports reviewed above. Given Romani et al.’s (1988) findings, prestimulus delta activity might directly modulate N1 latency, and inversely modulate N1 and P2 amplitudes. As their study employed a slow counting task, it was uncertain what effect prestimulus delta would have on RT performance, if any at all. Prestimulus theta may show a direct association with the component latencies of the N1 (Romani et al., 1988), and perhaps also P2, N2, and P3 (Lazzaro et al., 2001). Despite conflicting evidence reported for P2 (Lazzaro et al., 2001), N1 and P2 amplitudes might be inversely 
modulated by prestimulus theta - the more common finding (Rahn \& Başar, 1993; Romani et al., 1988). Prestimulus theta may also be inversely associated with N2 amplitudes, and directly with RT performance (Lazzaro et al., 2001). No predictions were made for either band regarding the P1 component, which does not appear to have been explored in relation to its possible prestimulus EEG determinants. There was no basis for making differential Go vs. NoGo predictions as each of the prior investigations reported target oddball responses or single stimulus paradigms only. However, due to theta's significantly greater implication in cognitive processes, this band might contribute to ERP Go/NoGo differences.

\section{Method}

\subsection{Participants}

Twenty undergraduate Psychology students (11 female, 9 male; 18 right-handed, 2 lefthanded), aged 17-30 years $(M=20.5, S D=3.1)$, volunteered and received course credit for their participation. All claimed normal hearing and abstinence from caffeine for at least four hours prior to testing, with no reports of prior severe head trauma, seizures, psychiatric illness, or recent psychoactive drug use. Written informed consent was obtained in accordance with a protocol approved by the University of Wollongong/Illawarra \& South East Sydney Area Health Service Human Research Ethics Committee.

\subsection{Physiological Recording}

A 16-bit A/D system (AMLAB II) recorded data between 0.03 and $35 \mathrm{~Hz}$ at a sample rate of $512 \mathrm{~Hz}$, with all electrode impedances below $5 \mathrm{~K} \Omega$. A 19 site cap with tin electrodes, complying with the international 10-20 system (Jasper, 1958), recorded continuous EEG referenced to physically-linked ears with a gain of 20,000. Vertical and horizontal electrooculograms (EOGs) were recorded with a gain of 5,000 from electrodes placed above and below the left eye, and beyond the outer canthus of each eye. Analysis was conducted off-line.

\subsection{Task and Procedure}

An equiprobable auditory Go/NoGo paradigm was utilised. The stimuli were 1000 and 
$1500 \mathrm{~Hz}$ tones, each of $50 \mathrm{~ms}$ duration, $5 \mathrm{~ms}$ rise/fall time, and a stimulus onset asynchrony (SOA) of 1,100 ms. Two stimulus blocks, each containing 150 tones (75 of each frequency in randomised order), were presented binaurally via circumaural headphones at $60 \mathrm{~dB}$ SPL. A button-press response with the dominant hand was required to the tone frequency designated as the 'target' (Go), with this frequency counterbalanced randomly across participants.

Upon arrival participants read an information sheet and provided informed consent before completing a screening questionnaire and having the physiological recording equipment fitted. Participants were seated in an air-conditioned, sound-attenuated booth during data collection, and were instructed to fixate on a small cross at the centre of a computer monitor (CRT) located approximately $1 \mathrm{~m}$ directly ahead of them to minimise eye artefact. Participants were requested to respond to their designated Go tone as quickly and accurately as possible.

\subsection{EEG Post-Processing and ERP Quantification}

The data were converted to Neuroscan format and epoched offline using Neuroscan software (Compumedics, Version 4.3). Artefact identification was completed via visual inspection of single trials, and those with muscular or other artefact were excluded from further analysis. The remaining data quantification was then completed within MATLAB® (The Mathworks, R14SP3) and EEGLAB (Version 6.01b; Delorme \& Makeig, 2004). The electrophysiological data were quantified for each participant separately, with the procedure applied individually for each frequency band (delta, 1-3 Hz; and theta, 4-7 Hz), and stimulus condition (Go and NoGo).

The initial processing protocol pertained only to the $\mathrm{Cz}$ data stream. First, $1 \mathrm{~s}$ epochs were derived, commencing 500 ms prior to stimulus onset, to allow epochs with incorrect responding to be identified. Each epoch was assessed for response accuracy, and those found to have omission errors to Go trials (RT > $500 \mathrm{~ms}$ ), or commission errors to NoGo trials, were excluded from further analysis. Prestimulus epochs of 500 ms duration were then derived for all accepted trials, and each was baselined across their duration. The data for each prestimulus epoch was reflected within the time domain, and a FFT decomposition was performed on the now $1 \mathrm{~s}$ equivalent data- 
epochs. The time-domain reflection technique was applied to improve the spectral resolution $(\Delta \mathrm{f}$ $=1 \mathrm{~Hz}$ ), and also to overcome the Gibbs phenomenon (Pan, 2001). The FFT decomposition involved the transformation of the reflected data to the frequency domain, the exclusion of each individual frequency bin falling outside the prescribed EEG band limits, and the subsequent return to the time domain via inverse-FFT. The resulting FFT-decomposed prestimulus Cz epochs for the specified EEG band were then sorted according to ascending level of activity. That is, the accepted prestimulus epochs were ordered according to the sum of their spectral amplitudes within the defined band. Importantly, this epoch sorting index was recorded for subsequent application.

The second quantification protocol was applied to each data stream from the nine inner electrodes (F3, Fz, F4, C3, Cz, C4, P3, Pz, and P4). First, the continuous raw EEG data were retrieved, epoched over the $1 \mathrm{~s}$ interval from $-500 \mathrm{~ms}$, and baselined across the $100 \mathrm{~ms}$ period immediately prestimulus. Accepted wide-band epochs were then re-ordered via the application of the EEG band-specific sorting index derived from the initial protocol. The upper and lower thirds of the sorted epochs were separately averaged to obtain ERPs for High and Low levels of prestimulus activity in the particular frequency band. The ERPs were then imported into Neuroscan and an automated function was utilised to detect component peaks within set latency ranges: P1, 25-140 ms; N1, 70-190 ms; P2, 100-270 ms; N2, 140-320 ms; and P3, 225-390 ms; with manual peak verification and adjustment conducted by an experienced ERP researcher.

\subsection{Statistical Analysis}

Over all accepted trials, prestimulus EEG activity at Cz was examined as a function of Stimulus (Go vs. NoGo) in a single-factor ANOVA. To assess the appropriate selection of High/Low prestimulus EEG trials, activity at Cz was examined separately for the delta and theta bands. These MANOVAs included Level (High vs. Low prestimulus FFT band amplitude) and Stimulus (Go vs. NoGo).

The grand mean (across accepted trials) ERP amplitude and latency effects were examined independently for five components (P1, N1, P2, N2, and P3). Several three-way MANOVAs were 
conducted, each incorporating factors of Stimulus (Go vs. NoGo), and both Sagittal (Frontal, Central, Parietal) and Lateral (Left, Midline, Right) topographic dimensions. The topographic dimensions included planned contrasts which, for the Sagittal factor, compared the frontal (F) versus parietal $(\mathrm{P})$ regions, and central $(\mathrm{C})$ versus the fronto-parietal regional mean $(\mathrm{F} / \mathrm{P})$. The Lateral contrasts compared the hemispheric activity in the left (L) versus right (R) hemisphere, and activity in the midline (M) versus the hemispheric mean (L/R). These contrasts allow efficient identification of regional effects within the 3 x 3 electrode array.

Amplitude and latency data for the ERPs for the High and Low levels of prestimulus EEG activity were analysed separately for each band and ERP component. The MANOVAs and topographic contrasts applied for the grand mean ERP analyses were used, with Level (High vs. Low prestimulus FFT band amplitude) as an additional factor. As the effects of prestimulus FFT amplitude were of interest here, only main effects or interactions involving Level are reported.

The influence of the prestimulus EEG activity on RT to Go stimuli was examined for the delta and theta bands via separate $F$ tests. The single factor of Level (High vs. Low prestimulus FFT band amplitude) was analysed.

Each analysis listed above is completely within-subject. For all analyses, Bonferroni type $\alpha$ adjustments were unnecessary, as all contrasts were planned and there were fewer of these than degrees of freedom for effect (Tabachnick \& Fidell, 2007). Each F test reported had $(1,19)$ degrees of freedom.

\section{Results}

\subsection{Prestimulus EEG Level}

Figure 1 illustrates the mean prestimulus spectral amplitudes at $\mathrm{Cz}$, with $1 \mathrm{~Hz}$ resolution, as derived from the FFT-decomposed epochs averaged across all accepted trials (panel a), and across those trials contributing to the ERPs for the High vs. Low prestimulus EEG investigations of the delta (panel b) and theta (panel c) bands. As seen in panel a, the mean frequency contributions for the grand mean accepted trials did not differ between stimulus conditions $\left(F=1.8, p=.198, \eta_{p}^{2}=\right.$ 
$.07)$.

A main effect of Level was found for both the delta $\left(F=786.3, p<.001, \eta_{p}{ }^{2}=.98\right)$, and theta $\left(F=172.4, p<.001, \eta_{p}{ }^{2}=.90\right)$ bands, as evident in panels $\mathrm{b}$ and $\mathrm{c}$ respectively. That is, the prestimulus epochs representing the High prestimulus EEG activity had greater mean spectral amplitude, compared to the Low, across their corresponding band frequencies. There was no effect of Stimulus, nor a Level $\times$ Stimulus interaction (all $F \leq 1.92, p \geq .18, \eta_{p}{ }^{2} \leq .09$ ). Together these establish the appropriate trial sampling to examine prestimulus EEG - ERP effects for each band assessed.

Figure 1 about here.

\subsection{Grand Mean ERPs - Go/NoGo Effects}

A one-way ANOVA over Stimulus showed that the overall number of accepted trials was comparable across conditions (Go: $M=112.2, S D=16.7$; NoGo: $M=114.1, S D=17.3 ; F=0.41$, $\left.p=.532, \eta_{p}{ }^{2}=.02\right)$. The grand mean Go and NoGo ERPs for each analysed site are illustrated in Figure 2, with ERP components identified at C3. Note that across all sites there are no prestimulus peaks, precluding continuing poststimulus ERP contamination. The rising negativity evident at the non-frontal sites was interpreted as recovery of the previous P3 component, with the non-frontocentral distribution excluding the CNV.

Figure 2 about here.

Of the positive components, P1 occurred at a mean latency of $52.8(S D=2.1)$ ms and was evident at all sites. With a mean latency of $172.9(S D=1.5) \mathrm{ms}, \mathrm{P} 2$ was distinguishable at most sites, and a prominent P3, with mean latency of $304.4(S D=2.6) \mathrm{ms}$, was visible at all sites. Of the negative components, a pronounced N1 $(M=105.6$; $S D=1.5 \mathrm{~ms})$ was evident at all sites, and N2 $(M=208.9 ; S D=3.0 \mathrm{~ms})$ was discernable at most sites. A one-way ANOVA over Stimulus showed that latencies for each positive and negative component were comparable across conditions (all $F \leq 2.18, p \geq .156, \eta_{p}^{2} \leq .10$ ).

The topographic distributions of the grand mean ERP amplitudes at the nine sites are 
illustrated in Figure 3. For ease of interpretation, ERP components found to have significant Stimulus $\times$ Topography interactions are indicated by a green box enclosing the corresponding headmaps.

Figure 3 about here.

A Stimulus $\times$ Sagittal $\times$ Lateral MANOVA indicated that P1 was uniformly distributed across the scalp, with variation between Go and NoGo responses failing to reach significance (all $\left.F \leq 3.93, p \geq .06, \eta_{p}^{2} \leq .17\right)$. Sagittally, $\mathrm{P} 2$ amplitudes were larger parietally $(\mathrm{F}<\mathrm{P}: F=18.39, p<$ $\left..001, \eta_{p}{ }^{2}=.49\right)$, and were greatest in the central region (C $>$ F/P: $\left.F=46.96, p<.001, \eta_{p}{ }^{2}=.71\right)$. Laterally, $\mathrm{P} 2$ was greater in the midline than the hemispheres $\left(\mathrm{M}>\mathrm{L} / \mathrm{R}: F=22.96, p<.001, \eta_{p}{ }^{2}=\right.$ .55), and a Sagittal $\times$ Lateral interaction revealed that $\mathrm{P} 2$ was greatest at the vertex $(M>L / R \times C>$ F/P: $\left.F=5.35, p=.032, \eta_{p}{ }^{2}=.22\right)$. A Stimulus $\times$ Lateral interaction indicated that the central P2 enhancement was greater in the left hemisphere for NoGo than Go stimuli (Go $<$ NoGo $\times \mathrm{L}>\mathrm{R} \times$ C $>$ F/P: $\left.F=12.99, p=.002, \eta_{p}{ }^{2}=.41\right)$. Sagittaly, $\mathrm{P} 3$ amplitude had a centro-parietal distribution $\left(\mathrm{F}<\mathrm{P}: F=28.61, p<.001, \eta_{p}{ }^{2}=.60\right.$, and $\left.\mathrm{C}>\mathrm{F} / \mathrm{P}: F=63.87, p<.001, \eta_{p}{ }^{2}=.77\right)$. Laterally P3 was greater in the midline compared to the hemispheric mean $\left(\mathrm{M}>\mathrm{L} / \mathrm{R}: F=34.66, p<.001, \eta_{p}{ }^{2}=\right.$ .65), and a Sagittal $\times$ Lateral interaction revealed that this midline enhancement was increased centro-parietally $\left(\mathrm{M}>\mathrm{L} / \mathrm{R} \times \mathrm{F}<\mathrm{P}: F=17.74, p<.001, \eta_{p}{ }^{2}=.48\right.$, and $\mathrm{M}>\mathrm{L} / \mathrm{R} \times \mathrm{C}>\mathrm{F} / \mathrm{P}: F=$ $\left.7.15, p=.015, \eta_{p}{ }^{2}=.27\right)$. A series of Stimulus $\times$ Sagittal and Stimulus $\times$ Sagittal $\times$ Lateral interactions showed that Go P3 amplitudes were enhanced parietally (Go $>$ NoGo $\times \mathrm{F}<\mathrm{P}: F=$ 76.63, $p<.001, \eta_{p}{ }^{2}=.80$ ), more so in the left hemisphere (Go $>$ NoGo $\times \mathrm{L}>\mathrm{R} \times \mathrm{F}<\mathrm{P}: \mathrm{F}=9.10$, $p=.007, \eta_{p}{ }^{2}=.32$ ), and in the midline (Go $>$ NoGo $\times \mathrm{M}>\mathrm{L} / \mathrm{R} \times \mathrm{F}<\mathrm{P}: F=5.54, p=.029, \eta_{p}{ }^{2}=$ .23), while NoGo P3 amplitude was greatest at the vertex (Go $<$ NoGo $\times \mathrm{M}>\mathrm{L} / \mathrm{R} \times \mathrm{C}>\mathrm{F} / \mathrm{P}: \mathrm{F}=$ 23.19, $p<.001, \eta_{p}{ }^{2}=.55$ ). This separation of the parietal Go P3, and anteriorisation of the frontocentral NoGo P3, is evident in both Figure 2 and Figure 3.

Sagittally, N1 amplitudes were larger in the frontal (F $>$ P: $F=39.63, p<.001, \eta_{p}^{2}=.68$ ) 
and central regions $\left(\mathrm{C}>\mathrm{F} / \mathrm{P}: \mathrm{F}=4.75, p=.042, \eta_{p}{ }^{2}=.20\right)$. A Stimulus $\times$ Sagittal interaction revealed that the frontal enhancement of N1 was greater for Go than NoGo stimuli (Go > NoGo $\times$ F $>$ P: $F=5.04, p=.037, \eta_{p}{ }^{2}=.21$ ), while a Stimulus $\times$ Sagittal $\times$ Lateral interaction indicated that this was somewhat larger in the right hemisphere (Go $>$ NoGo $\times \mathrm{L}<\mathrm{R} \times \mathrm{F}>\mathrm{P}: F=4.22, p=.054$, $\eta_{p}{ }^{2}=.18$ ). $\mathrm{N} 2$ amplitudes appeared relatively positive in the context of surrounding peaks (see Figure 2). Sagittal contrasts showed that N2s were larger (more negative) in the frontal region (F $>$ P: $\left.F=36.55, p<.001, \eta_{p}{ }^{2}=.66\right)$ and relatively reduced (less negative) in the central region $(\mathrm{C}<$ F/P: $F=51.25, p<.001, \eta_{p}^{2}=.73$ ), indicating a strong frontal topography (see Figure 3). Sagittal $\times$ Lateral interactions indicated that this strong frontal topography was increased in the midline (M $>\mathrm{L} / \mathrm{R} \times \mathrm{F}>\mathrm{P}: F=11.10, p=.003, \eta_{p}{ }^{2}=.37 ;$ and $\mathrm{M}>\mathrm{L} / \mathrm{R} \times \mathrm{C}<\mathrm{F} / \mathrm{P}: F=5.01, p=.037, \eta_{p}{ }^{2}=.21$ respectively). A Stimulus $\times$ Sagittal interaction also reached significance, indicating that the frontal N2 enhancement was greater for Go than NoGo (Go $>$ NoGo $\times$ F $>$ P: $F=5.73, p=.027$, $\left.\eta_{p}^{2}=.23\right)$

\subsection{ERP Effects of Prestimulus EEG Levels}

No less than 27 epochs contributed to the ERPs for each of the High vs. Low prestimulus EEG levels. Again a one-way ANOVA over Stimulus showed that the number of accepted trials was comparable across Go $(M=38.1, S D=5.7)$ and NoGo $(M=38.7, S D=5.8)$ conditions $(F=$ $\left.0.38, p=.547, \eta_{p}^{2}=.02\right)$.

\subsubsection{High vs. Low prestimulus delta.}

For each of the ERP components investigated, Level $\times$ Stimulus MANOVAs indicated that ERP latency showed no significant variation between the High/Low prestimulus delta levels (all $F$ $\leq 3.38, p \geq .082, \eta_{p}{ }^{2} \leq .15$ ), and no interactions were found (all $F \leq 2.86, p \geq .107, \eta_{p}{ }^{2} \leq .13$ ).

Figure 4 illustrates the topographic distributions of the High vs. Low prestimulus delta ERP amplitudes at nine sites. Coloured boxes are again used to highlight those ERP components found to show a significant effect and/or interaction(s). Red boxes enclose maps with a 
prestimulus delta main effect of Level, blue encloses those with Level $\times$ Topography interactions only, and black encloses those with the combination of a main effect and topographic interaction(s).

Figure 4 about here.

A Level $\times$ Stimulus $\times$ Sagittal $\times$ Lateral MANOVA indicated that there was no main effect of Level for P1 $\left(F=3.48, p=.078, \eta_{p}{ }^{2}=.15\right)$, and although a Level $\times$ Sagittal $\times$ Lateral interaction revealed a central-right P1 enhancement for High compared to Low prestimulus delta (High > Low $\times \mathrm{L}<\mathrm{R} \times \mathrm{C}>\mathrm{F} / \mathrm{P}: F=8.96, p=.007, \eta_{p}{ }^{2}=.32$ ), none of the remaining higher-order interactions reached significance (all $F \leq 2.05, p \geq .168, \eta_{p}{ }^{2} \leq .10$ ). In P2, of the Level $\times$ Stimulus $\times$ Sagittal $\times$ Lateral MANOVA contrasts conducted, only a main effect of Level was found (High $>$ Low: $F=$ 26.80, $p<.001, \eta_{p}^{2}=.59$ ), with P2 amplitude enhancement seen for High prestimulus delta (all other $F \leq 2.52, p \geq .129, \eta_{p}{ }^{2} \leq .12$ ). Similarly, a main effect of Level was found in P3 (High > Low: $F=11.13, p=.003, \eta_{p}{ }^{2}=.37$ ), again with High prestimulus delta associated with increased amplitudes. A Level $\times$ Stimulus $\times$ Sagittal $\times$ Lateral interaction approached significance in P3, suggesting that the parietal-midline P3 enhancement was somewhat greater with High prestimulus delta for Go than NoGo stimuli (Go $>$ NoGo $\times$ High $>$ Low $\times$ M $>$ L/R $\times$ F $<$ P: $F=4.34, p=.051$, $\left.\eta_{p}^{2}=.19\right)$

The Level $\times$ Stimulus $\times$ Sagittal $\times$ Lateral MANOVA showed a main effect of Level in N1 where High prestimulus delta was associated with significantly diminished N1 amplitudes (High < Low: $F=11.15, p=.003, \eta_{p}{ }^{2}=.37$ ); while higher-order interactions revealed that this reduction was greatest at the vertex (High $<$ Low $\times \mathrm{M}>\mathrm{L} / \mathrm{R} \times \mathrm{C}>\mathrm{F} / \mathrm{P}: F=12.54, p=.002, \eta_{p}{ }^{2}=.40$ ), and that the central reduction was somewhat greater for NoGo than Go responses (Go $<$ NoGo $\times$ High $<$ Low $\times$ C $>$ F/P: $\left.F=4.07, p=.058, \eta_{p}{ }^{2}=.18\right)$. In N2, the Level $\times$ Stimulus $\times$ Sagittal $\times$ Lateral MANOVA contrasts revealed an amplitude reduction with High prestimulus delta (High < Low: F $\left.=18.57, p<.001, \eta_{p}{ }^{2}=.49\right)$, particularly at the vertex $($ High $<$ Low $\times \mathrm{M}>\mathrm{L} / \mathrm{R} \times \mathrm{C}>\mathrm{F} / \mathrm{P}: F=$ 
4.88, $p=.04, \eta_{p}{ }^{2}=.20$ ); no other higher-order interactions were found (all $F \leq 2.76, p \geq .113, \eta_{p}{ }^{2} \leq$ $.13)$.

A one-way ANOVA over Level revealed that mean reaction time performance showed only non-significant variation between High $(M=296.8, S D=43.6 \mathrm{~ms})$ and Low $(M=298.4, S D$ $=43.2 \mathrm{~ms})$ prestimulus delta trials $\left(F=0.18, p=.676, \eta_{p}^{2}=.01\right)$.

\subsubsection{High vs. Low prestimulus theta.}

The Level $\times$ Stimulus MANOVAs applied to each of the ERP components assessed showed that ERP latencies were comparable across prestimulus theta levels (all $F \leq 0.38, p \geq .545$, $\eta_{p}{ }^{2} \leq .02$ ), and no Level $\times$ Stimulus interactions were found (all $F \leq 2.26, p \geq .149, \eta_{p}{ }^{2} \leq .11$ ).

Topographic distributions of the High vs. Low prestimulus theta component amplitudes at nine sites are displayed in Figure 5. Colour-coded boxes border those component distributions found to show interaction effects. Here, blue indicates a Level $\times$ Topography interaction, orange identifies a Level $\times$ Stimulus interaction, and grey signifies a Level $\times$ Stimulus $\times$ Topography interaction.

\section{Figure 5 about here.}

In P1 amplitude, Level $\times$ Stimulus $\times$ Sagittal $\times$ Lateral MANOVA contrasts revealed that there were no prestimulus theta level effects $\left(F=1.30, p=.268, \eta_{p}{ }^{2}=.06\right)$, nor interactions (all $F \leq$ 2.15, $\left.p \geq .159, \eta_{p}{ }^{2} \leq .10\right)$. The Level $\times$ Stimulus $\times$ Sagittal $\times$ Lateral MANOVA contrasts in P2 revealed no main effect of Level $\left(F=0.11, p=.746, \eta_{p}{ }^{2} \leq .01\right)$, however, a Level $\times$ Sagittal interaction was found such that Low prestimulus theta was associated with a relative parietal enhancement (High $<$ Low $\left.\times \mathrm{F}<\mathrm{P}: F=10.97, p=.004, \eta_{p}{ }^{2}=.37\right)$. In P3, the MANOVA contrast of Level showed a main effect approaching significance $\left(F=4.04, p=.059, \eta_{p}^{2} \leq .17\right)$, while a Level $\times$ Sagittal interaction reached significance, indicating that Low prestimulus theta was associated with a relative parietal P3 enhancement (High $<$ Low $\times \mathrm{F}<\mathrm{P}: F=5.69, p=.028, \eta_{p}{ }^{2}=$ .23). A Level $\times$ Stimulus interaction also reached significance indicating that Go P3 was 
somewhat increased, but NoGo P3 was reduced with Low prestimulus theta (Go $<$ NoGo $\times$ High $>$ Low: $\left.F=6.23, p=.022, \eta_{p}^{2}=.25\right)$.

There was no main effect of Level for either N1 or N2 amplitude (both $F \leq 0.15, p \geq .701$, $\left.\eta_{p}{ }^{2}=.01\right)$. The MANOVA contrasts revealed a significant Level $\times$ Stimulus $\times$ Sagittal $\times$ Lateral interaction in N1: with Low theta, relative right-central N1 activity was reduced in Go and enhanced in NoGo (Go $<$ NoGo $\times$ High $<$ Low $\times \mathrm{L}<\mathrm{R} \times \mathrm{C}>$ F/P: $\left.F=5.05, p=.037, \eta_{p}{ }^{2}=.21\right)$. In N2, a Level $\times$ Stimulus interaction was significant: low prestimulus theta was also associated with a NoGo N2 enhancement (Go $<$ NoGo $\times$ High $<$ Low: $F=5.34, p=.032, \eta_{p}{ }^{2}=.22$ ).

The one-way ANOVA over Level showed that there was no significant variation in the mean RT performance between High $(M=296.4, S D=42.6 \mathrm{~ms})$ and Low $(M=295.0, S D=40.5$ ms) prestimulus theta $\left(F=0.11, p=.745, \eta_{p}^{2}=.01\right)$.

\section{Discussion}

As shown in Figure 1, the prestimulus spectral amplitudes of the $1 \mathrm{~Hz}$ narrow frequency bands did not differ between Go/NoGo stimuli across the accepted trials (panel a), or the trials selected based on their High/Low levels of prestimulus delta (panel b), or theta (panel c). The appropriate separation of trials having High/Low prestimulus activity in the delta and theta bands was also confirmed (refer Figure 1, panels b and c). Importantly, these results indicate that any stimulus-specific effects obtained here as a function of delta and theta are due to the differential manipulation of prestimulus activity within that band, and not a general frequency difference between Go and NoGo conditions.

\subsection{Latency and Reaction Time Effects}

Differential prestimulus activity in the delta and theta bands failed to produce variations in ERP latencies and reaction time. This suggests that, at least within this paradigm, prestimulus low-frequency EEG activity is not a determining factor of response timing. However, as this appears to be the first study to utilise an equiprobable task, these results need verification. Future 
investigations might also benefit by incorporating paradigms of increasing difficulty, as the lack of RT variation could be due to ceiling effects in our simple equiprobable task for the young adult population sampled.

\subsection{Amplitude Effects}

The Go/NoGo ERPs (Figures 2 and 3) show the typical fronto-central N1, centro-parietal P2, and frontal N2 distributions reported for the equiprobable paradigm (Barry, 2009). The clear separation of the parietal P3b and fronto-central P3a was also evident for the Go and NoGo distributions respectively, and is consistent with the Late Positive Complex (LPC) subcomponent literature regarding this paradigm (Barry \& Rushby, 2006).

\subsubsection{High vs. Low prestimulus delta.}

Prestimulus delta activity appears to be important in determining all components assessed, regardless of stimulus type. Prestimulus delta activity directly affected the positivity of the components - positive amplitudes were enhanced, and negative amplitudes reduced, with increased delta level. This was apparent as a main effect for each component except P1, where a regional effect occurred. Importantly, Figure 4 shows that similar effects were obtained in the response components to both the Go and NoGo stimuli, although there were some minor (nonsignificant) regional effects in NoGo N1 and Go P3.

\subsubsection{High vs. Low prestimulus theta.}

Prestimulus theta level had no effect on P1, however, several interactions with topography and/or stimulus condition produced a differential pattern of effects across the remaining components. Across stimulus conditions, parietal P2 and P3 amplitudes were inversely modulated by prestimulus theta. A complex higher-order interaction suggests that prestimulus theta directly modulated Go, and inversely modulated NoGo regional N1 responses. This is difficult to understand, and may indicate differential effects in N1 subcomponents; clearly this needs further investigation. Theta inversely modulated NoGo N2 and Go P3, but directly modulated NoGo P3. Together, these findings implicate prestimulus theta activity as a determinant of ERP amplitude 
outcomes, having a predominantly inverse influence. Notably, this differed for Go and NoGo conditions, suggesting a stimulus-specific and processing-related influence.

\subsection{Integration with Previous Findings}

Of the speculative associations derived in the Introduction from a diverse literature, the inverse relationship between prestimulus delta activity and N1 amplitude was fully confirmed, while the inverse relationship with P2 amplitude was contradicted. Partial confirmation of the inverse relationship between prestimulus theta activity and the N1, P2, and N2 amplitudes was also found via topographic and/or stimulus-type interactions.

Each of our speculations were based on limited findings, with the present study differing in many dimensions from those reviewed. For example, Romani et al. (1988) investigated only the combined influence of prestimulus delta and theta activity, and both Romani et al. (1988) and Rahn and Başar (1993) assessed the N1-P2 peak-to-peak amplitudes; we assessed these bands and components individually. Romani et al. (1988) employed a slow counting task during which the participants laid with their eyes closed, and Lazzaro et al. (2001) assessed between-subjects effects for an adolescent (11-17 years) AD/HD sample when compared to their matched controls for rare target oddball stimuli. In contrast, our Go/NoGo task was delivered to seated participants with eyes open, we investigated the within-subjects effects for a healthy young adult sample, and assessed both stimulus conditions within an equiprobable paradigm. Finally, the developmental and meditation papers reported EEG findings across a broader, and not necessarily task-related period, and also reported simultaneous fluctuations across several EEG bands. We selectively averaged the trials based on their task-related prestimulus activity within individual bands. Given the number of differences in population, paradigm, procedure, and quantification methods, the discrepancies between results are not surprising. This could be addressed in future investigations of prestimulus EEG - ERP determinants by incorporating paradigmatic and/or other relevant manipulations.

The pattern of findings across the two low-frequency bands emphasises the importance of 
the EEG activity occurring immediately-prestimulus, and hence supports the contribution of the ongoing EEG in ERP genesis. Even so, this support for the phase-reset model does not rule out indirect contributions from evoked activity, and hence we cannot attribute all the effects to either of the proposed mechanisms of ERP genesis. However, our results are broadly consistent with prior studies, which have found that effects in the early components tend to favour the phase-reset model, while the later components are more likely to reflect evoked activity (Barry, 2009).

\subsection{Prestimulus Low-Frequency EEG and Cognitive Functionality}

Delta activity immediately preceding stimulus onset directly affected P1, but more strongly inversely affected N1 amplitudes. Each of these components is thought to indicate sensory processing (Näätänen \& Picton, 1987) and perceptual resource allocation (Kok, 1997). Moreover, these components are typically reported to be modulated by attention (Kok, 1997; Näätänen \& Picton, 1987). An inverse relationship between prestimulus delta and N2 was found. This component is largely associated with response inhibition (Smith et al., 2008), but also response conflict (Lavric et al., 2004). P3 amplitudes, commonly associated with target identification and event categorisation, each thought to be sensitive to attention and working memory processes (Kok, 1997, 2001), were directly modulated by prestimulus delta. Interestingly, these amplitude modulations did not differ between stimulus conditions, consistent with the exogenous attention effects. However, inhibition as indicated by N2 amplitude (Smith et al., 2008), and event categorisation as indexed by P3 amplitude (Kok, 1997, 2001), typically differ between stimulus conditions, and thus our results suggest that prestimulus delta has little or no impact on endogenous processing. The general pattern of prestimulus delta influence on ERP amplitudes support the poststimulus delta band interpretations discussed in the Introduction, specifically regarding the exogenous processes of signal detection, stimulus matching, and decision making (Başar et al., 2000, 2001; Başar-Eroglu et al., 1992; Başar-Eroglu \& Demiralp, 2001; Başar-Eroglu et al., 2001).

The stimulus-specific nature of the prestimulus theta - ERP amplitude modulations 
strongly implicates this band in cognitive processing outcomes, and associates low prestimulus theta with improved processing. Although there were stimulus-specific prestimulus theta effects for N1, the complex nature of the interaction found is difficult to interpret, and further work should aim to address this. More importantly, prestimulus theta activity inversely affected NoGo N2 responses. This finding is consistent with the N2 link to inhibition (Smith et al., 2008) and response conflict (Lavric et al., 2004), and may underlie the typical increase in NoGo N2 observed in comparison to Go N2 amplitudes (Smith et al., 2008). Of particular interest here, the prestimulus theta - P3 amplitude relationship showed a differential pattern across the stimulus conditions. Go P3 was inversely related, while NoGo P3 was directly related, to prestimulus theta activity. Functionally, the P3 is most associated with target identification involving both attention and memory processing (Kok, 1997, 2001), compatible with the inverse Go P3 modulation found. However, P3 amplitude has also been reported to partly indicate cognitive and motor inhibition processes, with the NoGo P3 typically increased compared to the Go P3 amplitudes in this respect (Smith et al., 2008). Therefore, our direct NoGo P3 finding can be accounted for by both the P3 inhibition interpretation and an increase in attention during event categorisation (Kok, 2001). Together these findings are broadly in agreement with the attention (Başar et al., 2000, 2001; Başar-Eroglu et al., 1992; Başar-Eroglu \& Demiralp, 2001; Başar-Eroglu et al., 2001) and memory (Klimesch, 1999) processing interpretations of theta band activity.

\subsection{Summary and Conclusion}

Given the functional importance of the delta and theta EEG bands regarding cognitive processing, it is essential to understand their influence on ERP component outcomes. We assessed the nature of the immediately-prestimulus EEG influences of these low-frequency bands individually, substantially extending the few prior investigations. Significant ERP amplitude modulations were found with our equiprobable auditory Go/NoGo paradigm, differentially implicating each band as determining factors in ERP amplitude and cognitive processing outcomes. In summary, prestimulus delta activity has a direct global effect on the positivity of the 
component amplitudes. Hence, low prestimulus delta produces greater sensory processing (N1), and response conflict and inhibition processing (N2), but reduced activation for event categorization (P3). Prestimulus theta produced more complex modulations of the component amplitudes, in a stimulus-specific fashion. Importantly, low prestimulus theta was associated with NoGo inhibition (N2), and both Go target identification and NoGo inhibition and event categorisation (P3).

This appears to be the first study to utilise a short inter-stimulus interval (ISI) equiprobable task to assess the low-frequency prestimulus EEG contributions to ERP component outcomes, and as such it is not free of limitations. One potential concern has been overcome in our methodology. That is, our lowest assessed frequency was $1 \mathrm{~Hz}$ (included in the delta band) and our prestimulus interval of 500 ms would not normally have captured this frequency in the FFT. To overcome this we employed a data-mirroring technique (Pan, 2001) which allowed us to conduct an FFT on the equivalent of $1 \mathrm{~s}$ of data, and which provided us with the $1 \mathrm{~Hz}$ resolution required to assess contributions of this frequency range. Another concern regards the potential contamination of the prestimulus EEG by ongoing responses to the preceding stimulus. Given our fixed 1,100 ms SOA, we chose to restrict our immediately-prestimulus interval to $500 \mathrm{~ms}$ in order to minimise the likelihood of ongoing ERP contamination. This prestimulus interval selection allowed for $600 \mathrm{~ms}$ of ERP responding prior to the prestimulus interval of the following stimulus. Inspection of the ERPs (Figure 2) shows no prestimulus peaks, but continuing recovery of the preceding P3. This rising prestimulus negativity has a non-fronto-central distribution, arguing against CNV contamination. Moreover, this CNV-like P3 component recovery seen in the prestimulus ERPs appears to have a frequency $<0.5 \mathrm{~Hz}$, and thus would not have contributed to the High/Low effects since our FFT-separated sorting bands did not include activity of that frequency (i.e., delta = 1-3 Hz; theta $=4-7 \mathrm{~Hz}$ ). While it is important to replicate the present findings in future investigations, further studies should expand this line of research by incorporating paradigms with long ISIs, and also with varying ISIs. Extending this investigation to assess the remaining EEG 
bands is also suggested, with this project presently underway in our lab in relation to prestimulus alpha and beta contributions. The strong results obtained here are very interesting, and suggest insights into the genesis of the ERP in cognitive processing tasks. Further, once the contributions from each EEG band are mapped out, applications across clinical populations could provide insight into the EEG mechanisms contributing to their associated cognitive deficits. 


\section{References}

Barry, R.J., 2009. Evoked activity and EEG phase resetting in the genesis of auditory Go/NoGo ERPs. Biological Psychology 80, 292-299.

Barry, R.J., Clarke, A.R., 2009. Spontaneous EEG oscillations in children, adolescents, and adults: Typical development, and pathological aspects in relation to AD/HD. Journal of Psychophysiology 23, 157-173.

Barry, R.J., Kirkaikul, S., Hodder, D., 2000. EEG alpha activity and the ERP to target stimuli in an auditory oddball paradigm. International Journal of Psychophysiology 39, 39-50.

Barry, R.J., Rushby, J.A., 2006. An orienting reflex perspective on anteriorisation of the P3 of the event-related potential. Experimental Brain Research 173, 539-545.

Başar, E., Başar-Eroglu, C., Karakaş, S., Schürmann, M., 2000. Brain oscillations in perception and memory. International Journal of Psychophysiology 35, 95-124.

Başar, E., Başar-Eroglu, C., Karakaş, S., Schürmann, M., 2001. Gamma, alpha, delta, and theta oscillations govern cognitive processes. International Journal of Psychophysiology 39, 241-248.

Başar, E., Başar-Eroglu, C., Rosen, B., Schutt, A., 1984. A new approach to endogenous eventrelated potentials in man: Relation between EEG and P300-wave. International Journal of Neuroscience 24, 1-21.

Başar, E., Stampfer, H. G., 1985. Important associations among EEG-dynamics, event-related potentials, short-term memory and learning. International Journal of Neuroscience 26, 161180.

Başar-Eroglu, C., Başar, E., Demiralp, T., Schürmann, M., 1992. P300-response: Possible psychophysiological correlates in delta and theta frequency channels. A review. International Journal of Psychophysiology 13, 161-179. 
Başar-Eroglu, C., Demiralp, T., 2001. Event-related theta oscillations: An integrative and comparative approach in the human and animal brain. International Journal of Psychophysiology 39, 167-195.

Başar-Eroglu, C., Demiralp, T., Schürmann, M., Başar, E., 2001. Topographical distribution of oddball ‘P300’ responses. International Journal of Psychophysiology 39, 213-220.

Cahn, B.R., Polich, J., 2006. Meditation states and traits: EEG, ERP, and neuroimaging studies. Psychological Bulletin 132, 180-211.

Delorme, A., Makeig, S., 2004. EEGLAB: An open source toolbox for analysis of single-trial EEG dynamics including independent component analysis. Journal of Neuroscience Methods 134, 9-21.

Intriligator, J., Polich, J., 1994. On the relationship between background EEG and the P300 eventrelated potential. Biological Psychology 37, 207-218.

Intriligator, J., Polich, J., 1995. On the relationship between EEG and ERP variability. International Journal of Psychophysiology 20, 59-74.

Jasper, H.H., 1958. The ten-twenty electrode system of the International Federation. Electroencephalography and Clinical Neurophysiology 10, 371-375.

Jervis, B.W., Nichols, M.J., Johnson, T.E., Allen, E., Hudson, N.R., 1983. A fundamental investigation of the composition of auditory evoked potentials. IEEE Transactions on Biomedical Engineering 30, 43-50.

Karakaş, S., Erzengin, Ö.U., Başar, E., 2000. A new strategy involving multiple cognitive paradigms demonstrates that ERP components are determined by the superposition of oscillatory responses. Clinical Neurophysiology 111, 1719-1732.

Klimesch, W., 1999. EEG alpha and theta oscillations reflect cognitive and memory performance: A review and analysis. Brain Research Reviews 29, 169-195. 
Klimesch, W., Sauseng, P., Hanslmayr, S., Gruber, W., Freunberger, R., 2007. Event-related phase reorganization may explain evoked neural dynamics. Neuroscience and Biobehavioral Reviews 31, 1003-1016.

Kok, A., 1997. Event-related-potential (ERP) reflections of mental resources: A review and synthesis. Biological Psychology 45, 19-56.

Kok, A., 2001. On the utility of P3 amplitude as a measure of processing capacity. Psychophysiology 38, 557-577.

Kolev, V., Rosso, O.A., Yordanova, J., 2001. A transient dominance of theta ERP component characterizes passive auditory processing: Evidence from a developmental study. NeuroReport 12, 2791-2796.

Kolev, V., Schürmann, M., 1992. Event-related prolongation of induced EEG rhythmicities in experiments with a cognitive task. International Journal of Psychophysiology 67, 199-213.

Lavric, A., Pizzagalli, D.A., Forstmeier, S., 2004. When 'go' and ‘nogo' are equally frequent: ERP components and cortical tomography. European Journal of Neuroscience 20, 2483-2488.

Lazzaro, I., Gordon, E., Whitmont, S., Meares, R., Clarke, S., 2001. The modulation of late component event related potentials by pre-stimulus EEG theta activity in ADHD. International Journal of Neuroscience 107, 247-264.

Min, B.-K., Busch, N.A., Debener, S., Kranczioch, C., Hanslmayr, S., Engel, A.K., Herrmann, C.S., 2007. The best of both worlds: Phase-reset of human EEG alpha activity and additive power contribute to ERP generation. International Journal of Psychophysiology 65, 58-68.

Näätänen, R. Picton, T., 1987. The N1 wave of the human electric and magnetic response to sound: A review and an analysis of the component structure. Psychophysiology 24, 375425.

Pan, C., 2001. Gibbs phenomenon removal and digital filtering directly through the Fast Fourier Transform. IEEE Transactions on Signal Processing 49, 444-448. 
Polich, J., 1997. On the relationship between EEG and P300: Individual differences, aging, and ultradian rhythms. International Journal of Psychophysiology 26, 299-317.

Rahn, E., Başar, E., 1993. Prestimulus EEG-activity strongly influences the auditory evoked vertex response: A new method for selective averaging. International Journal of Neuroscience 69, 207-220.

Romani, A., Callieco, R., Cosi, V., 1988. Prestimulus spectral EEG patterns and the evoked auditory vertex response. Electroencephalography and Clinical Neurophysiology 70, 270272.

Sauseng, P., Klimesch, W., Gruber, W.R., Hanslmayr, S., Freunberger, R., Doppelmayr, M., 2007. Are event-related potential components generated by phase resetting of brain oscillations? Neuroscience 146, 1435-1444.

Segalowitz, S.J., Santesso, D.L., Jetha, M.K., 2010. Electrophysiological changes during adolescence: A review. Brain and Cognition 72, 86-100.

Smith, J.L., Johnstone, S.J., Barry, R.J., 2008. Movement-related potentials in the Go/NoGo task: The P3 reflects both cognitive and motor inhibition. Clinical Neurophysiology 119, 704714.

Spencer, K.M., Polich, J., 1999. Poststimulus EEG spectral analysis and P300: Attention, task, and probability. Psychophysiology 36, 220-232.

Stampfer, H.G., Başar, E., 1985. Does frequency analysis lead to better understanding of human event related potentials. International Journal of Neuroscience 26, 181-196.

Tabachnick, B.G., Fidell, L.S., 2007. Using multivariate statistics. Boston: Pearson Education, Inc. Yordanova, J., Kolev, V., 1998. Single-sweep analysis of theta frequency band during an auditory oddball task. Psychophysiology 35, 116-126. 


\section{Figure Legends}

Figure 1. Mean prestimulus EEG spectra at Cz for accepted Go (green) and NoGo (red) trials. a. Grand mean across accepted trials, b. High and Low prestimulus delta (1-3 Hz), c. High and Low prestimulus theta (4-7 Hz). Mean spectral amplitudes differed significantly for the High/Low levels of prestimulus delta and theta as indicated above their constituent frequencies in panels b and c, respectively. $* * * p<.001$.

Figure 2. Grand mean ERP waveforms for accepted Go (black) and NoGo (grey) trials at each site analysed. The components labelled at C3 (middle-left) were examined.

Figure 3. Topographic plots of the grand mean (across accepted trials) amplitudes for each ERP component of interest at the nine sites analysed (F3, Fz, F4, C3, Cz, C4, P3, Pz, P4; locations indicated on the top-left headmap). The mean across conditions, and separate distributions for the Go and NoGo stimulus conditions, are displayed. For each ERP component, the scale is identified at the base of its column. A green box indicates a significant Stimulus $\times$ Topography interaction $(p<.05)$

Figure 4. Topographic plots of each ERP component for the High and Low prestimulus delta (1-3 $\mathrm{Hz}$ ) waveforms at nine analysed sites (F3, Fz, F4, C3, Cz, C4, P3, Pz, P4; locations indicated on the top-left headmap). The scale for each ERP component is identified at the base of its column. The mean across conditions, and separate distributions for the Go and NoGo stimulus conditions, are displayed for each level of prestimulus delta activity assessed. For the headmaps enclosed, a coloured box indicates a significant $(p<.05)$ main effect of Level (red), a Level $\times$ Topography interaction (blue), or their joint occurrence (black). 
Figure 5. Topographic plots of each ERP component for the High and Low prestimulus theta (4-7 $\mathrm{Hz}$ ) waveforms at nine analysed sites (F3, Fz, F4, C3, Cz, C4, P3, Pz, P4; locations as indicated on the top-left headmap). The scale for each ERP component is identified at the base of its column. The mean across conditions, and separate distributions for the Go and NoGo stimulus conditions, are displayed for each prestimulus theta level examined. Coloured boxes indicate significant $(p<$ .05) Level $\times$ Topography (blue), Level $\times$ Stimulus (orange), and Level $\times$ Stimulus $\times$ Topography (grey) interactions. 
Figure 1.

a

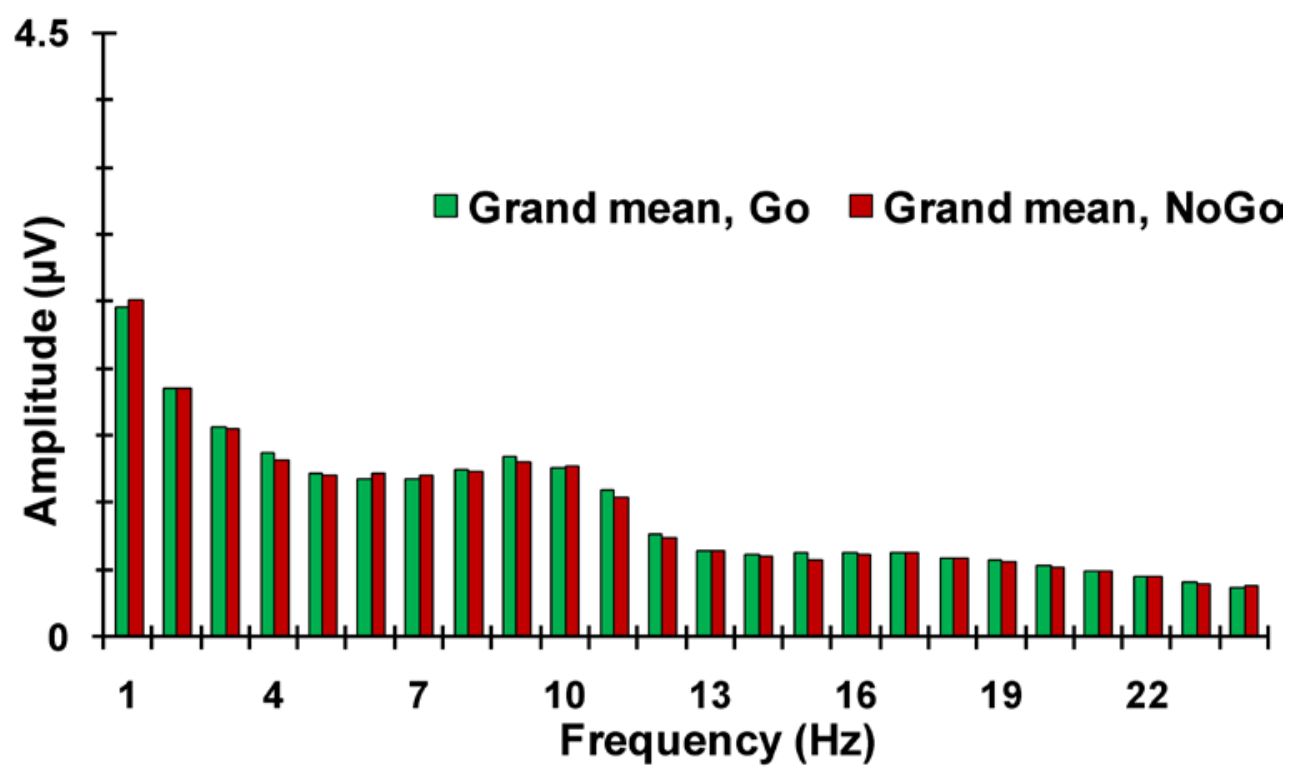

b

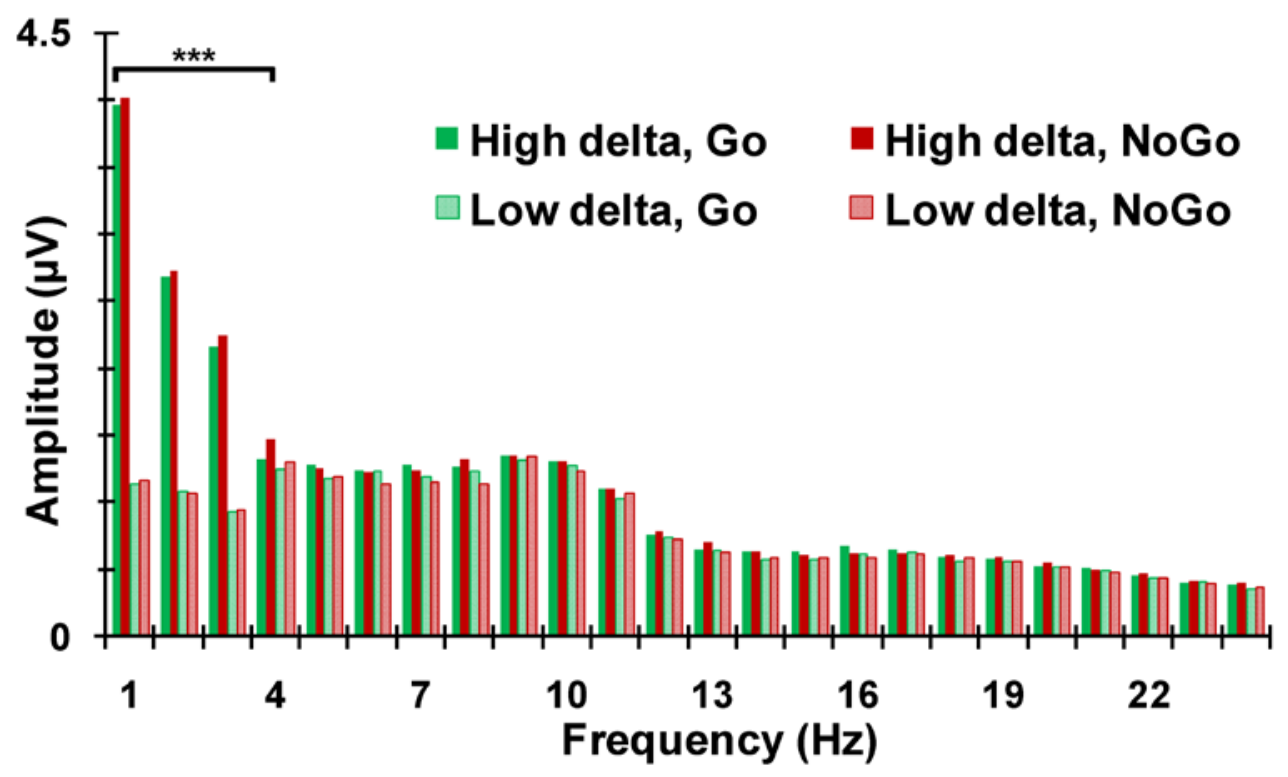

C

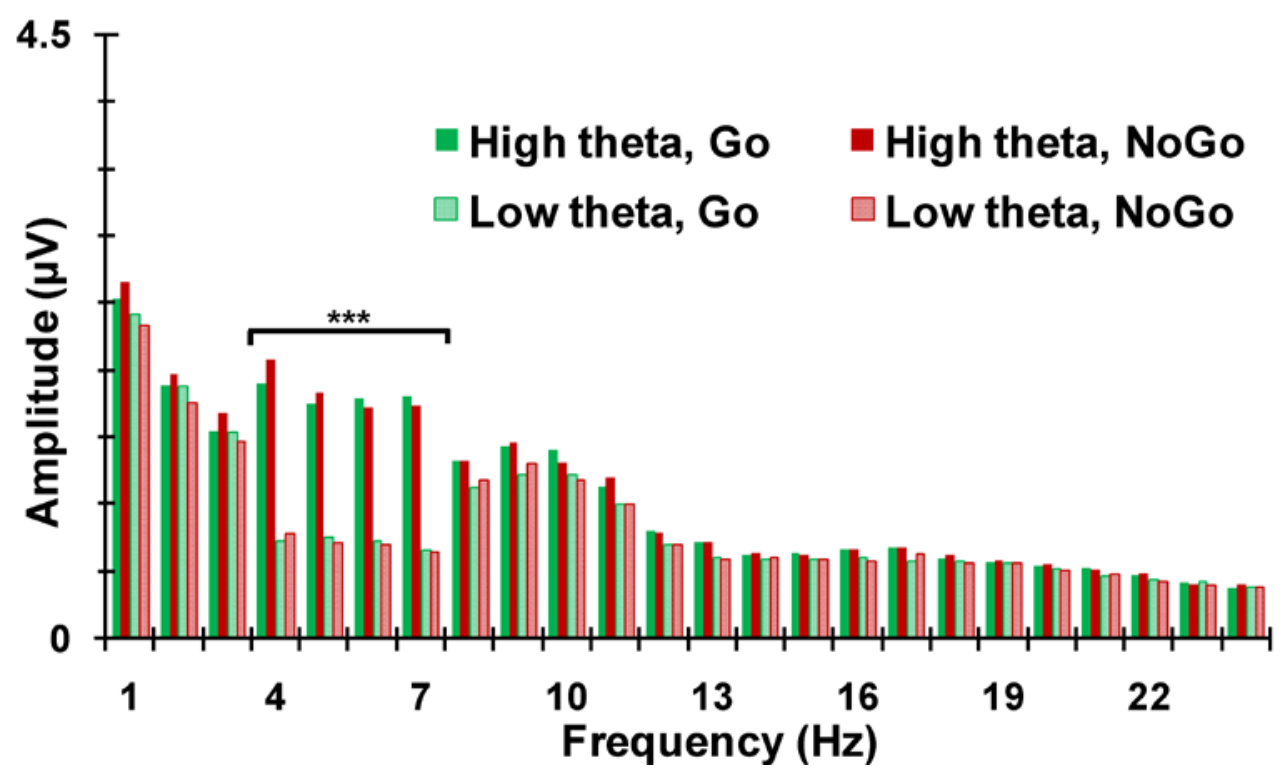


Figure 2.
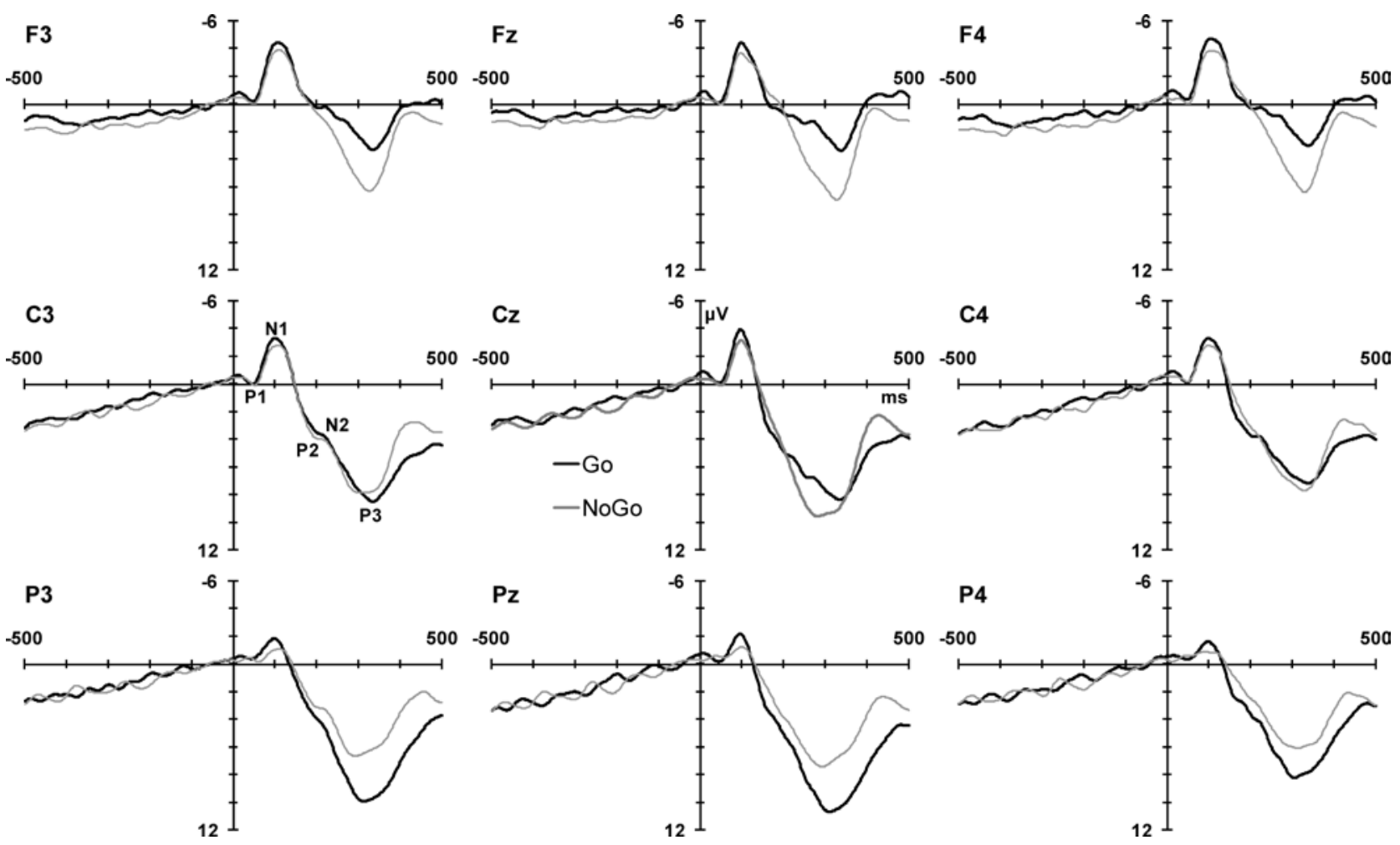

Figure 3.
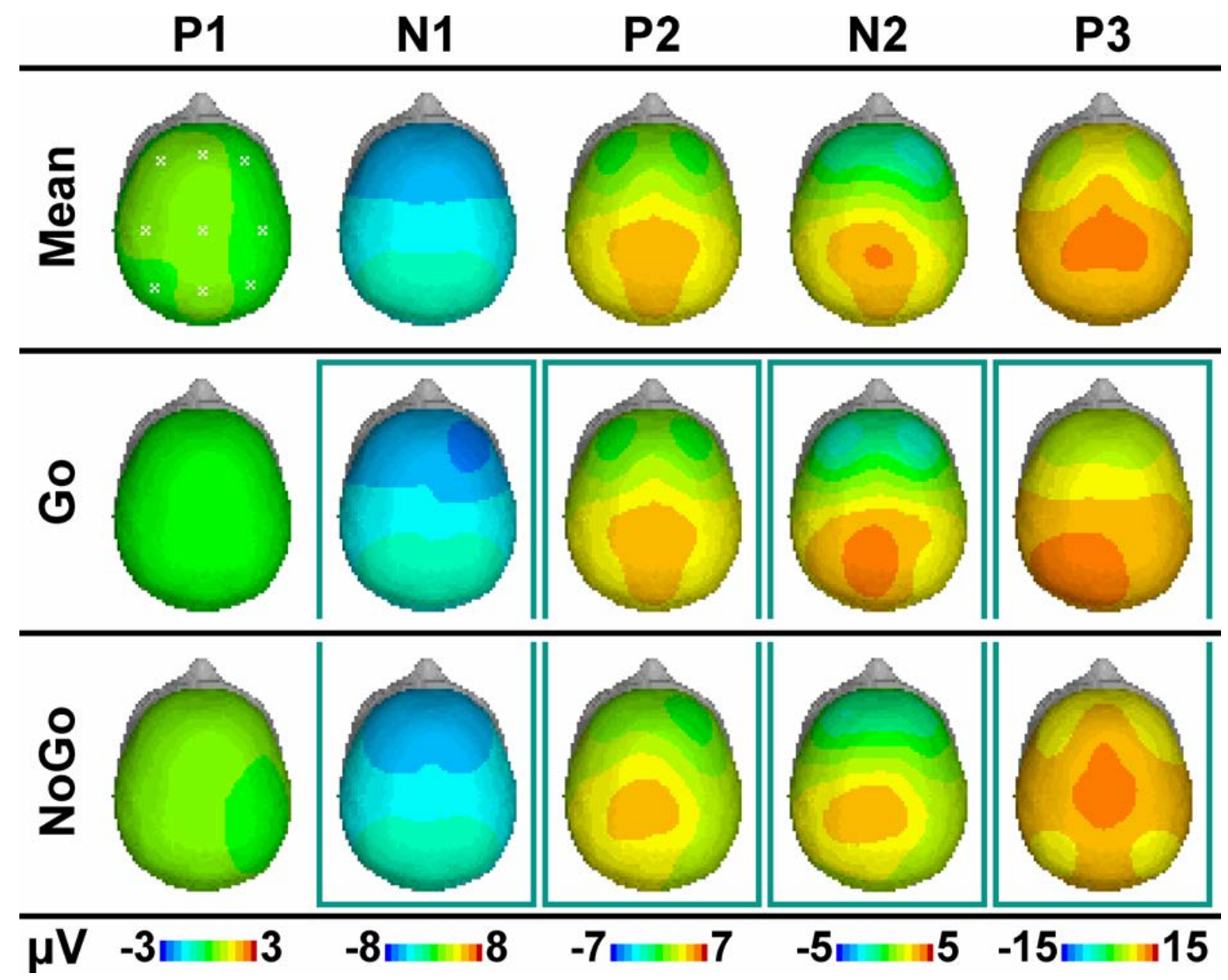
Figure 4.
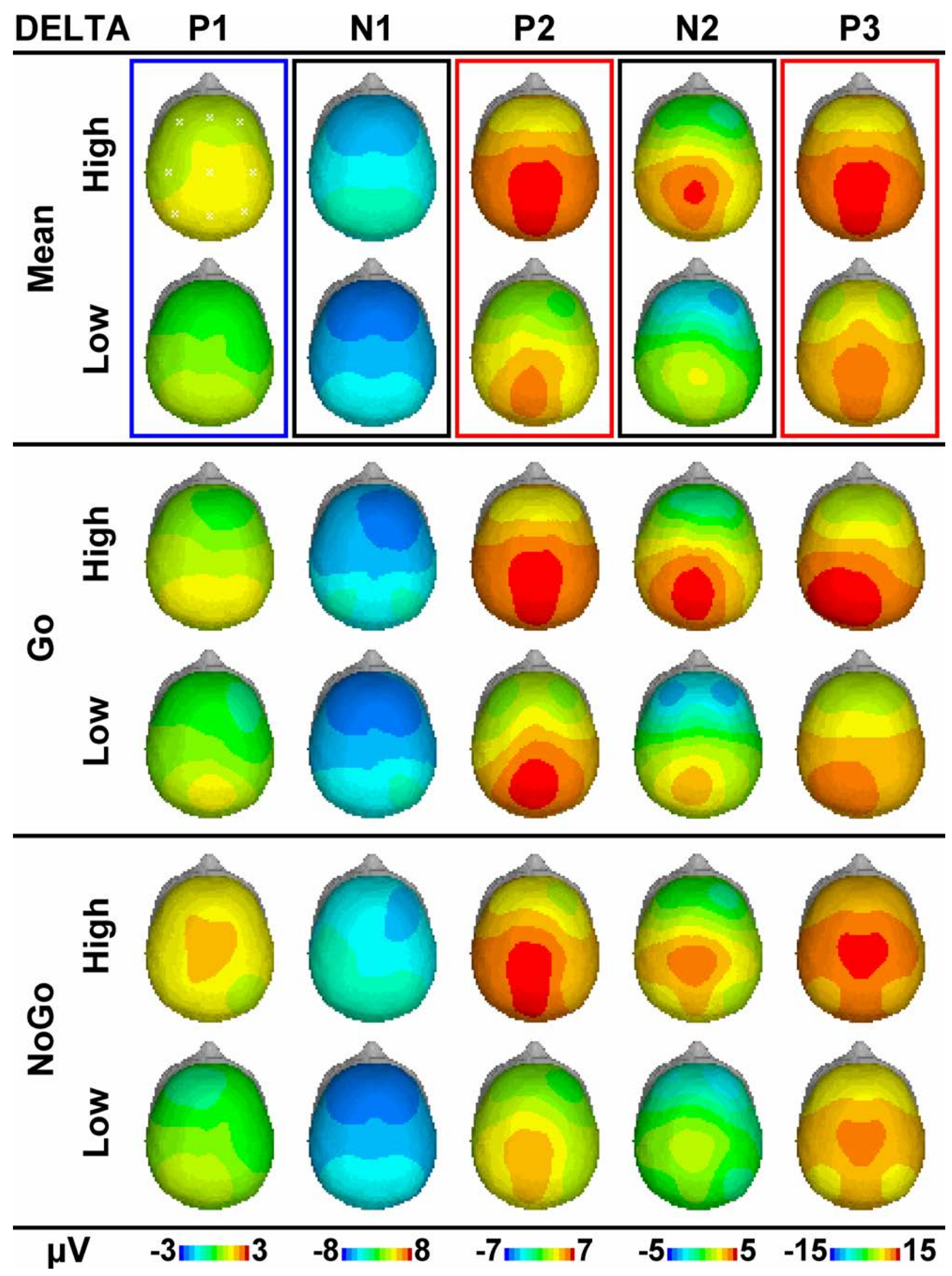
Figure 5.
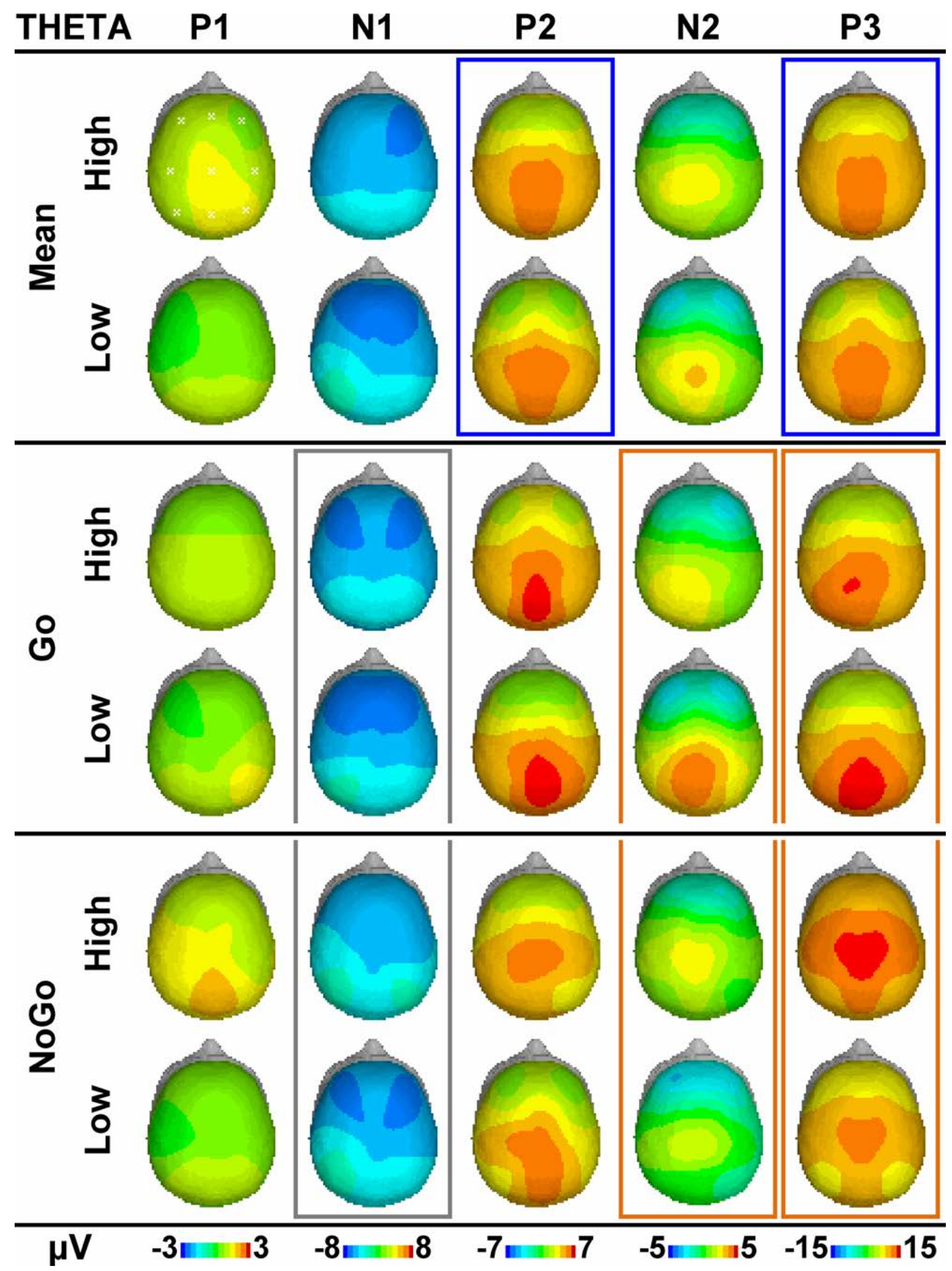